\title{
Deep Learning, Ubiquitous and Toy Computing Minitrack
}

\author{
Patrick C. K. Hung \\ Faculty of Business and Information Technology, University of Ontario Institute of Technology, Canada \\ patrick.hung@uoit.ca \\ Shih-Chia Huang \\ Department of Electronic Engineering, National Taipei University of Technology, Taiwan \\ schuang@ntut.edu.tw \\ Sarajane Marques Peres \\ School of Arts, Sciences and Humanities, University of Sao Paulo, Brazil \\ sarajane@usp.br
}

\begin{abstract}
The goal of this minitrack is to present both novel and industrial solutions to challenging technical issues as well as compelling smart application use cases. This minitrack will share related practical experiences to benefit the reader and will provide clear proof that deep learning technologies are playing an ever-increasing important and critical role in supporting ubiquitous and toy computing applications - a new cross-discipline research topic in computer science, decision science, and information systems.
\end{abstract}

\section{Introduction}

The pervasive nature of digital technologies as witnessed in industry, services and everyday life has given rise to an emergent, data-focused economy stemming from many aspects of human individual and ubiquitous applications. The richness and vastness of these data are creating unprecedented research opportunities in many fields including urban studies, geography, economics, finance, entertainment, and social science, as well as physics, biology and genetics, public health and many other smart devices. In addition to data, text and machine mining research, businesses and policymakers have seized on deep learning technologies to support their decisions and proper growing smart application needs.

As businesses build out emerging hardware and software infrastructure, it becomes increasingly important to anticipate technical and practical challenges and to identify best practices learned through experience in this research area. Deep learning employs software tools from advanced analytics disciplines such as data mining, predictive analytics, text, and machine learning based on a set of algorithms that attempt to model high-level abstractions in data by using multiple processing layers with complex structures or non-linear transformations.

At the same time, the processing and analysis of deep learning applications present methodological and technological challenges. Further deep learning applications are advantaged by a rise in sensing technologies as witnessed in both the number of sensors and the rich diversity of sensors ranging from cell phones, personal computers, and health tracking appliances to Internet of Things (IoT) technologies designed to give contextual, semantic data to entities in a ubiquitous environment that previously could not contribute intelligence to key decisions and smart devices. Recently deep learning technologies have been applied to toy computing. Toy computing is a recently developing concept which transcends the traditional toy into a new area of computer research using ubiquitous technologies. A toy in this context can be effectively considered a computing device or peripheral called Smart Toys.

There are two research papers presented in this minitrack. The first paper "Twitter Connections Shaping New York City" by Sobolevsky et al. presents a novel way of constructing a spatial social network based on such data to analyze its structure and evaluate its utility for delineating urban neighborhoods. The second paper "Perceived Innovativeness and Privacy Risk of Smart Toys in Brazil and Argentina" by Fantinato et al. studies Brazilian and Argentinian consumers' perceived innovativeness, risks and benefits of smart toys and their purchase intention toward such toys. 